\section{Significance and relationship between DJ-1 gene and survivin gene expression in laryngeal squamous cell carcinoma}

\author{
Z. Shen, ${ }^{1,2}$ Y. Ren, ${ }^{2}$ D. Ye, ${ }^{1,2}$ J. Guo, ${ }^{2}$ \\ C. Kang, ${ }^{2}$ H. Ding ${ }^{2,3}$ \\ 'Department of Otorhinolaryngology- \\ Head and Neck Surgery, Lihuili Hospital \\ of Ningbo University; \\ ${ }^{2}$ Ningbo University School of Medicine; \\ ${ }^{3}$ Department of Pathology, Lihuili \\ Hospital of Ningbo University, China
}

\section{Abstract}

This study aimed at exploring the correlation between DJ-1 gene and survivin gene in laryngeal squamous cell carcinoma by analyz ing their gene expression levels and their relationship with clinicopathologic parameters. The expression of DJ-1 gene and survivin gene in 82 laryngeal carcinoma tissues from patients and 82 negative surgical margin tissue samples were detected by immunohistochemistry, respectively. The correlation of their expression levels and patients' clinical parameters were then analyzed by Pearson correlation analysis. The positive detection rates of DJ-1 and survivin in laryngeal carcinoma tissues were $71.95 \%$ and $60.98 \%$, which were higher than those of the normal control that were $29.27 \%$ and $0.00 \%$, respectively $(\mathrm{P}<0.01)$. The positive detection rates of DJ-1 and survivin were found associated with tumor stages $(\mathrm{P}<0.05)$, but not with lymph node metastasis. The DJ-1 gene expression level was related to cell differentiation $(\mathrm{P}<0.05)$. Finally, a positive correlation between DJ-1 and survivin gene expression in laryngeal carcinoma was found. The overall survival rate of patients was $51.2 \%$, and disease-free survival (DFS) was $39.0 \%$. DFS in DJ-1 negativeexpression group was $87.0 \%$, and $20.3 \%$ in DJ1 positive-expression group. The negative expression of DJ-1 was associated with a shorter mean patient DFS time $(44.643 \pm 1.417$ months), whereas positive expression of DJ-1 was associated with a longer mean DSF time $(25.943 \pm 1.297$ months). DJ-1 and survivin play a vital role in the occurrence and development of laryngeal carcinoma. DJ-1 may promote the carcinogenesis of laryngeal cells by up-regulating the survivin gene expression.

\section{Introduction}

Laryngeal carcinoma is a common malignant tumor. Globally speaking, it is the second incidence rate among head and neck cancers, and the $11^{\text {th }}$ among all human cancers. ${ }^{1}$ The major pathological type of laryngeal cancers is squamous cell carcinoma, accounting for $99 \%$ of the laryngeal malignant tumors. The laryngeal carcinoma incidence rate has an obvious trend of increase in recent years, but the mechanism of its occurrence and development is still unclear.

DJ-1 gene is a new found mitogen dependence oncogene. Early study showed that the mutation of DJ-1 gene might cause Parkinsonism disease. ${ }^{2}$ Recent studies indicate that DJ- 1 is closely related to the occurrence, progress and prognosis of the malignant tumors. ${ }^{3-6}$

Survivin is a kind of inhibitors of apoptosis proteins (IAPs). Because of its special biological structure and characteristics, survivin plays a vital role in the occurrence and progress of most malignant tumors by participating in inhibiting apoptosis, interrupting mitosis and angiogenesis, and reducing chemoradiation sensitivity. ${ }^{7-10}$

The functional studies of DJ-1 and survivin on carcinogenesis have shown a possible relationship between DJ-1 and phosphatase and tensin homolog (PTEN), a famous tumor suppressor gene, as well as PTEN and survivin. ${ }^{11-14}$ However, the correlation between DJ-1 gene and survivin gene in the laryngeal squamous cell carcinoma (LSCC) has not been reported.

Our previous study found that both mRNA and protein levels of DJ-1 and survivin were suppressed by DJ-1-small interference RNA. ${ }^{15}$ The result demonstrates a possible linkage between DJ-1 and survivin. To explore the clinical significance of DJ-1 and survivin, this study compared DJ-1 and survivin expressions in LSCC tissues and negative surgical margin tissues by immunohistochemistry and confirmed their correlation in carcinogenesis. We hope to assess the possibility of using them as prognostic markers in LSCC, to improve laryngeal cancer diagnosis and provide a new theoretical basis for decision-making.

\section{Materials and Methods}

\section{Materials}

A total of 82 cancer samples and 82 negative surgical margin tissue samples was collected from patients with LSCC who received surgery between 2006 and 2010 at Li Huili Hospital, Ningbo, China. Informed consent was obtained from all subjects. The Human Research Ethics
Correspondence: Dr. Zhisen Shen, Department of Otorhinolaryngology-Head and Neck Surgery, Lihuili Hospital of Ningbo University, Ningbo, 315000 , China.

Tel. +86.13.906687216 - Fax: +86.574 .87392232 . E-mail:szs7216@sina.com

Key words: laryngeal carcinoma, DJ-1 gene, survivin gene, diagnosis.

Acknowledgements: this work was supported by grants from the Ningbo Social Developmental Key Research Project (No. 2008C50019) and the K.C. Wong Magna Fund in Ningbo University, China.

Contributions: ZS, conception, design, data acquisition, analysis and interpretation; YR, DY, conception, design, data acquisition, article drafting; JG, critical manuscript revision; CK, conception and design; HD, data acquisition.

Conflict of interest: the authors declare no potential conflict of interest.

Received for publication: 16 October 2010 . Accepted for publication: 22 February 2011.

This work is licensed under a Creative Commons Attribution 3.0 License (by-nc 3.0).

(C) Copyright Z. Shen et al., 2011

Licensee PAGEPress, Italy

European Journal of Histochemistry 2011; 55:e9 doi:10.4081/ejh.2011.e9

Committee in Li Huili Hospital approved all aspects of the study. None of these patients had received prior treatment. The diagnosis was confirmed by two pathologists. The extent of tumor at the time of operation was classified according to the tumor-node-metastasis (TNM) staging system of the International Union Against Cancer. The case numbers of T1 T4 were $22,17,27$, and 16, respectively. Among them, 54 cases had no lymph node metastasis (N0). For clinical stages from I to IV, their numbers were $17,16,34$, and 15 , respectively. The numbers of well differentiation, moderately differentiation, and poor differentiation were 29, 36 , and 17 , respectively. Patients were scheduled for physical examination, laryngofiberscopy, and chest X-ray or CT scan every month during the first year after surgery, then every 3 months for 2 years, and every 6 months thereafter. Follow-up focused specifically on recurrence (local and distant), disease-free survival (DFS), and overall survival. There have been 40 patients died and 10 recurrences during our follow-up by December 2010.

\section{Antibodies}

The rabbit antihuman survivin and DJ-1 polyclonal antibody for immunohistochemistry 
were purchased from Zhongshan Golden Bridge Biotechnology Company (Beijing, China) and Abcam Biotechnology Company (Cambridge, MA, USA), respectively. Tetrazolium (BCIP/NBT) alkaline phosphatase color development kit was from Boster Biotechnology Company (Wuhan, China).

\section{Immunohistochemistry}

The procedures included follow steps: dewaxed and hydrated; rinsed with water; repaired antigens under pressure by citric acid buffer for $2.5 \mathrm{~min}$; rinsed with water; hydrogen peroxide solution was added to remove endogenous peroxidase; incubated at room temperature for $10 \mathrm{~min}$; rinsed with phosphate buffer solution (PBS); incubated with primary antibody at $4^{\circ} \mathrm{C}$ over night; rinsed with PBS; second antibody was added and incubated for 15 min; rinsed with PBS; cells were colored by Diaminobenzidine (DAB) Horseradish Peroxidase Color Development Kit (Maixin Biotechnology Co., Fujian, China). At last, cells were after stained by hematoxylin (Maixin).

The assessment of positive stain for DJ-1 protein: under high power field of microscopy, 100 tumor cells were counted; and total of five fields were investigated. According to the positive rate, the expression of DJ-1 was divided into two groups: negative expression (positive rate $<5 \%$ ) and positive expression (positive rate $>5 \%) .{ }^{16}$ The assessment of positive stain for survivin protein, the positive-cell rate and stain degree of positive cells were divided into different levels. The score criterion of positive cell rate was: 0 (cytoplasm without being dyed), 1 (1-25\%), 2 (26\%-50\%), 3 (51-75\%), and 4 (76-100\%). The score criterion for stain degree of positive cells was: 0 (negative), 1 (weak stain), 2 (moderate stain), and 3 (strong stain). If the sum of two scores was more than 4 , the expression of survivin was considered as positive. ${ }^{17}$

\section{Western blot analysis}

Lysates from tissue samples were prepared using radioimmunoprecipitation (RIPA) buffer [10 mM Tris-HCl (pH 7.4), $150 \mathrm{mM} \mathrm{NaCl}, 1 \%$ NP-40, $1 \mathrm{mM}$ EDTA, 0.1\% SDS, and $1 \mathrm{mM}$ dithiothreitol]. After centrifugation at 14,000 rpm for $15 \mathrm{~min}$ at $4^{\circ} \mathrm{C}$, the supernatants were collected. Proteins were resolved by SDS-polyacrylamide gel electrophoresis, and then transferred to polyvinylidene fluoride (PVDF) membranes (Millipore Corporation, Bedford, MA, USA). The membranes were blocked with $5 \%$ defatted milk powder for $2 \mathrm{~h}$ at room temperature, then incubated with rabbit anti-human DJ-1 antibody or anti-human survivin antibody (Santa Cruz Biotechnology, CA, USA) at $4^{\circ} \mathrm{C}$ overnight and subsequently incubated for $1 \mathrm{~h}$ with alkaline phosphatase conjugated goat anti-rabbit antibody (Boster). The expression of $\beta$-actin (Sigma-Aldrich, Saint Louis, M0, USA) was used as a normalization control for protein loading. The immunoblots were visualized by use of a 5-bromo-4-chloro-3-indolylphosphate/nitroblue.

\section{Statistical analysis}

Statistical analysis was performed using Statistical Analysis System (SAS) software 6.0 (SAS Institute Inc., Cary, NC, USA). The difference of DJ-1 or survivin positive detection rates between laryngeal carcinoma tissues and normal tissues, and their relationship with clinical parameters were analyzed by $\chi^{2}$-test. The correlation between DJ-1 expression and survivin expression in laryngeal carcinoma tissues was analyzed by Pearson correlation analysis. The correlation between the expression of different markers and patient survival was done by Kaplan-Meier analysis. The logrank test was applied to test whether the correlation was statistically significant. Multivariate survival analysis was performed with the Cox regression analysis. The level of significance was set at $\mathrm{P}<0.05$.
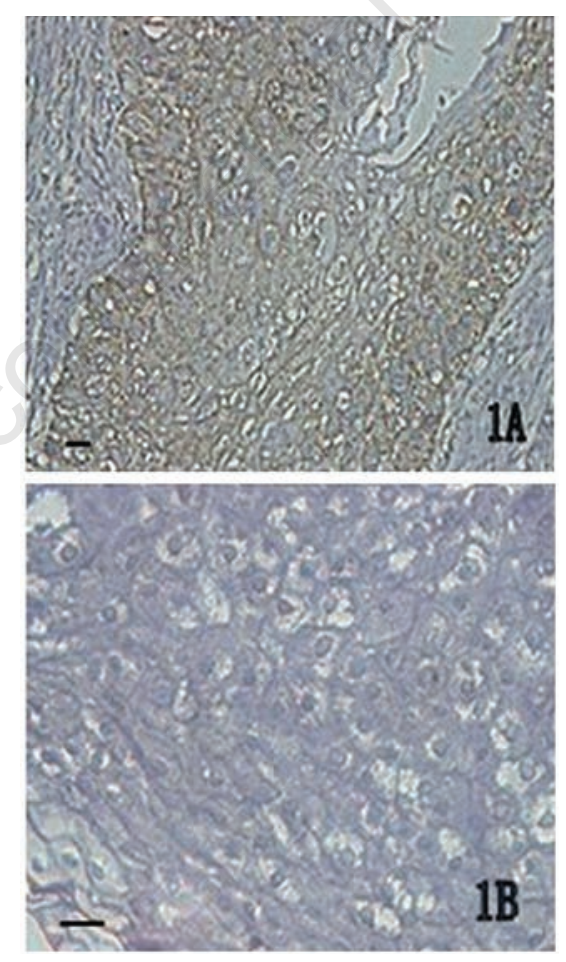

Figure 1. Immunohistochemistry analysis of DJ-1 protein in laryngeal carcinoma tissues (A) and normal laryngeal mucous tissues (B). A, high differentiated squamous cell carcinoma. The DJ-1 protein, which was mainly found in cytoplasm, was stained as yellowish-brown ( $\times 20)$; B, normal laryngeal mucous tissues. There was no DJ-1 expression in cytoplasm $(\times 40)$. Scale bar: $100 \mu \mathrm{m}$.

\section{Results}

\section{DJ-1 and survivin expressions in} laryngeal carcinoma tissues and normal laryngeal mucous tissues

The DJ-1 protein, which was mainly stained in cytoplasm, was observed in cancer cells, but not in normal cells (Figure 1). Similar to DJ-1, survivin protein also stained in yellowishbrown and found in cytoplasm of cancer cells (Figure 2). The positive detection rate of DJ-1 protein in laryngeal carcinoma tissues was $71.95 \%$, while $29.27 \%$ in normal laryngeal mucous tissues $(\mathrm{P}<0.01)$. The survivin expression was only found in cancer tissues. The positive detection rate of survivin protein in laryngeal carcinoma tissues was $60.98 \%(\mathrm{P}<0.01$, comparing with normal tissues).

\section{Relationship between DJ-1 expres- sion or survivin expression in laryn- geal carcinoma tissues and clinical parameters}

By analyzing the positive detection rates of

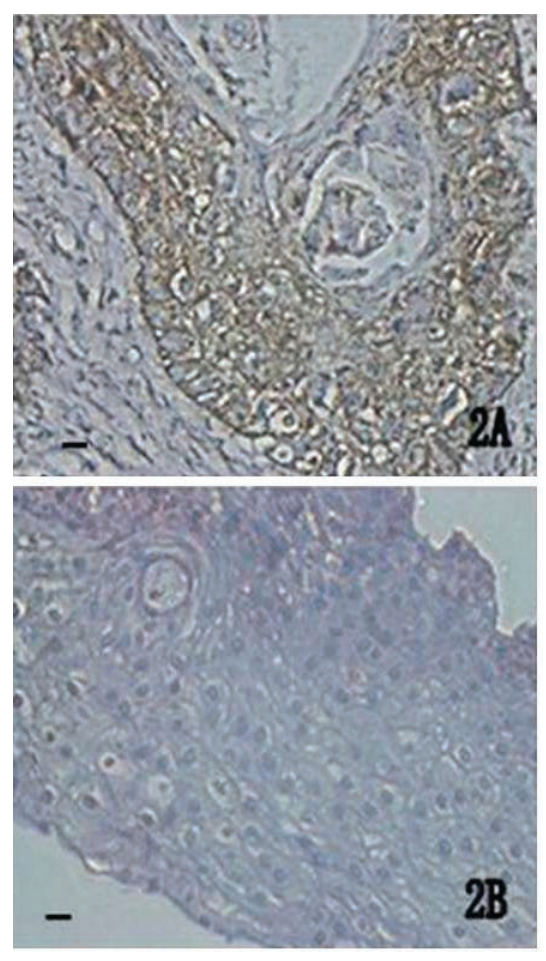

Figure 2. Immunohistochemistry analysis of survivin protein in laryngeal carcinoma tissues (A) and normal laryngeal mucous tissues (B). A, well differentiated squamous cell carcinoma. The survivin protein, which was mainly fund in cytoplasm, was stained as yellowish-brown $(\times 20)$; B, normal laryngeal mucous tissue. There was no survivin expression in cytoplasm $(\times 20)$. Scale bar: $100 \mu \mathrm{m}$. 
DJ-1 protein and survivin protein, we found that the positive detection rates of DJ-1 and survivin expression were associated with tumor stages $(\mathrm{P}<0.05)$, but not with lymph node metastasis (Table 1). DJ-1 expression, but not survivin expression, was related to cell differentiation $(\mathrm{P}<0.05$, Table 2$)$.

\section{Correlation of DJ-1 expression and survivin expression in laryngeal cancer tissues}

Among 82 laryngeal cancer cases, 44 were both DJ-1 and survivin positive expressed, and 17 both negative expressed (Table 3). Pearson correlation analysis indicated that a positively correlation between DJ-1 and survivin expression in laryngeal carcinoma was found $(\mathrm{r}=0.4079)$.

\section{Western blot analysis of DJ-1 and survivin}

As shown in Figure 3, compared with negative surgical margin tissue samples, increased DJ-1 and survivin expression in LSCC were detected by Western blot. Survivin did not expressed in negative surgical margin tissue. Their over expression was associated with poorer cell differentiation, higher pTNM pathologic staging and T staging.

\section{Survival analysis}

The overall survival rate of 82 patients was
51.2\%, and DFS was $39.0 \%$. Using univariate Kaplan-Meier survival estimation with logrank comparison, we found that DFS in DJ-1 negative-expression group was $87.0 \%$, and $20.3 \%$ in DJ-1 positive-expression group. The negative expression of DJ-1 was associated with a shorter mean patient DFS time (44.643 \pm 1.417 months), whereas positive expression of DJ-1 was associated with a longer mean DSF time (25.943 \pm 1.297 months; Figure 4). And DFS was correlated with T stage, pTNM pathologic staging, N stage, and degree of tumor differentiation $(\mathrm{P}<0.05)$. Multivariate survival analysis was performed with the Cox proportional hazards model. It showed that only pTNM pathologic staging, DJ1 and survivin expressions were independent predictor for LSCC patient survival $(\mathrm{P}<0.05$; hazard ratio: 4.087, 15.817 and 5.111, respectively); while $\mathrm{T}$ stage, $\mathrm{N}$ stage and degree of tumor differentiation were not $(\mathrm{P}>0.05)$.

The relationship between overall survival and DJ-1 expression, survivin expression and clinicopathologic variables is shown in Table 4. Just like the DFS, only DJ-1 expression, survivin expression and pTNM pathologic staging were the predictor parameter for overall survival. We also analyzed the subgroup according to DJ-1/surviving expression, and found that DJ-1+/surviving+ subgroup had the poorest prognosis, while the DJ-1/survivin- subgroup had the best prognosis (Figure 5 and Table 4).

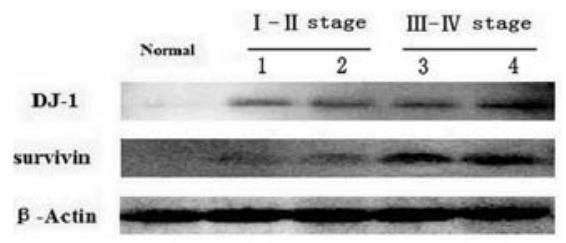

Figure 3. Western blot results of DJ-1 and survivin expression.

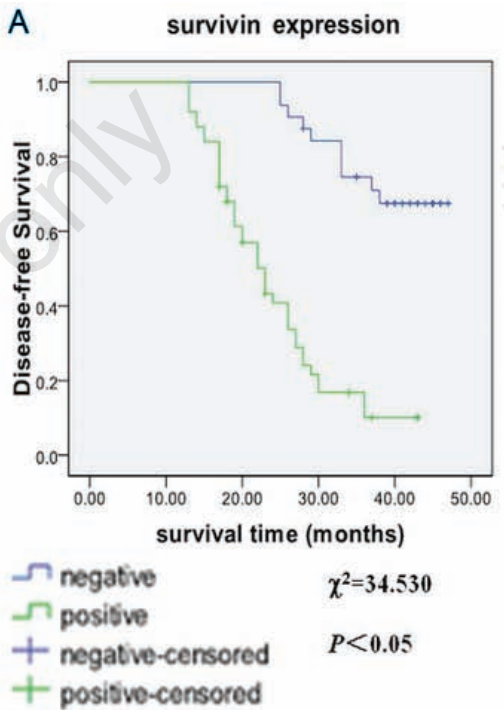

B

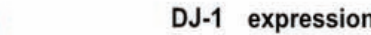

Table 1. Relationship between DJ-1 or survivin expressions and clinical parameters.

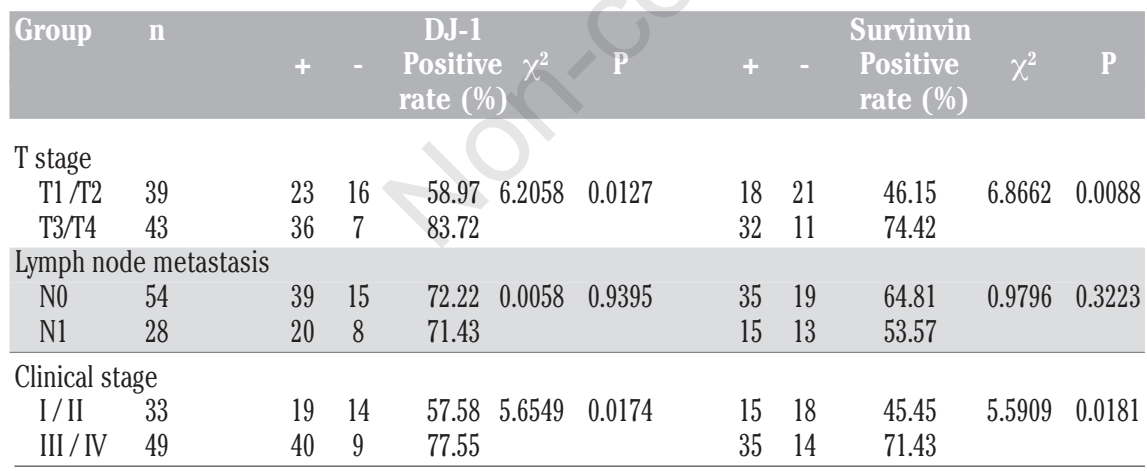

Table 2. Relationship between DJ-1 or survivin expressions and pathologic differentiation.

\begin{tabular}{lccccccccccc} 
Groulp & $\mathrm{n}$ & \multicolumn{4}{c}{$\begin{array}{c}\text { DJ-1 } \\
\text { Positive }\end{array}$} & $\chi^{2}$ & $\mathrm{P}$ & + & $\begin{array}{c}\text { Survinvin } \\
\text { Positive } \\
\text { rate (\%) }\end{array}$ & $\chi^{2}$ & $\mathrm{P}$ \\
$\begin{array}{l}\text { Well- } \\
\text { differentiated }\end{array}$ & 29 & 16 & 13 & 55.17 & 6.25900 .0124 & 13 & 16 & 44.83 & 4.91680 .0266 \\
$\begin{array}{l}\text { Moderate, } \\
\text { poor } \\
\text { differentiated }\end{array}$ & 53 & 43 & 10 & 81.13 & & 37 & 16 & 69.81 & \\
\end{tabular}

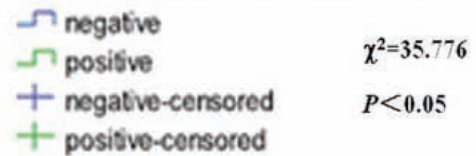

Figure 4. Kaplan-Meier analysis of the significance of $\mathrm{DJ}-1$ and survivin expression and clinicopathologic parameters in predicting patient survival. A, disease-freesurvival curve between different $\mathrm{DJ}-1$ expressions. B, disease-free-survival curve between different survivin expressions. 


\section{Discussions}

Studies have shown that the occurrence and development of malignant tumor are closely related to the over expression of some oncogenes and apoptosis inhibitory factors. As a mitogen dependent oncogene, DJ-1 has become a research focus in the last years. A proteomics testing found that breast cancer cell lines SUM-44 secreted DJ-1 protein and that the DJ-1 expression and its antibodies in breast cancer were more than those in noncancer cases. The measurement of DJ-1 antibodies in peripheral blood was thought be a useful marker of detection the occurrence and development of breast cancer. ${ }^{5}$ MacKeigan et $a l .{ }^{6}$ reported that in primary lung cancer patients, the recurrence rate of patients with high-level of DJ-1 obviously was higher than that with low-level of DJ-1. DJ-1 in cervical tissues was obviously increased and might be used as a cervical cancer progression marker. ${ }^{18}$ A positive relationship of DJ-1 with tumor invasion and metastasis was found in hepatocellular carcinoma, and DJ-1 was also highlighting an important role in chemoresistance.${ }^{19}$ Landon et al. ${ }^{20}$ identified several ligand binding hot spots for DJ-1, and one in the dimer interface might be bound with pharmacological chaperone. These hot spots were considered providing novel starting points for drug discovery efforts. Considering its effect in oncogenesis, the roles of DJ-1 in the occurrence, development and prognosis of laryngeal cancer need to be cleared.

In this study, we found that DJ-1 expression level in laryngeal cancer tissues was significantly higher than that in normal mucosa (Figure 1). Its positive detection rates were associated with tumor stages and cell differentiation (Table 1-2). The high level of DJ-1 expression might indicate worse $\mathrm{T}$ stage, pTNM pathologic stage and differentiation. In Western blot analysis, this tendency could be presented as well. Contradictory to previous studies, there was no significant difference between DJ-1 expression of groups with and without lymph node metastasis. So we did not find the evidence of the role of DJ-1 in LSCC metastasis. Concerning survival, DJ-1 positive expression significantly affected the overall survival and DFS of patients with LSCC, and it served as an independent predictor related to DFS after surgery for laryngeal cancer. These results indicate that DJ-1 may play an important role in the development of laryngeal cancer, and a potential prognostic parameter for LSCC.

As the strongest apoptosis inhibitory factor, survivin was involved in the regulation of cellular proliferation and angiogenesis in cancer. $^{21}$ Wang et al..$^{22}$ found that survivin expressed in laryngeal squamous cell carcinoma, but did not express in normal laryngeal mucous tissue. In our study, survivin was also found highly expressed in laryngeal cancer. In addition, the Western blot analysis confirmed the result. The level of its expression was associated with several clinicopathologic parameters (Figure 2; Table 1); high level might predict worse prognosis as an independent predictor (Figure 4). All result implied that survivin was a useful prognostic marker for LSCC.

In our previous study, we found that the expression levels of these two genes in laryngeal cancer Hep-2 cells were consistent. ${ }^{15}$ In this study, this linkage was studied using laryngeal cancer tissues. The results showed that the DJ-1 gene expression was positively correlated to the survivin gene expression (Table 3). The same phenomenon has been found in breast, ${ }^{5}$ cervical ${ }^{18}$ and liver cancer. ${ }^{23}$ Studies have shown that DJ-1 is a regulatory of PTEN, a recognized tumor suppressor gene, which promotes the PI3K/Akt/mTOR pathways so as to cause the constant proliferation and growth of cells. ${ }^{34}$ Recently, Sitaram et al. ${ }^{24}$ found that DJ-1 gene regulated the expression of PTEN through PI3K-AKT/PKB pathways. Survivin expression is negatively correlated to PTEN expression in cancers. Carter et al. ${ }^{11}$

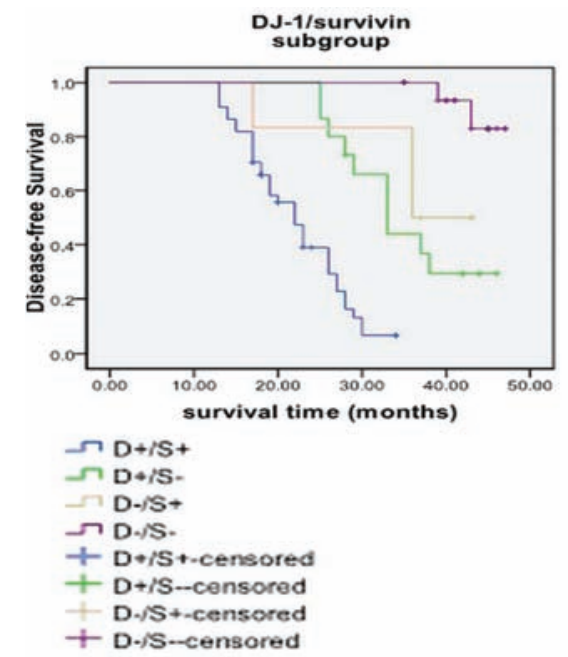

Figure 5. Survival curves of the subgroups analyzed by Kaplan-Merier analysis.

Table 3. Correlation of DJ-1 expression and survivin expression in laryngeal cancer tissues.

\begin{tabular}{lcc} 
Survivin & & DJ-1 \\
& + & - \\
+ & 44 & 6 \\
- & 15 & 17 \\
\hline
\end{tabular}

Table 4. Univariate and multivariate analysis of overall survival.

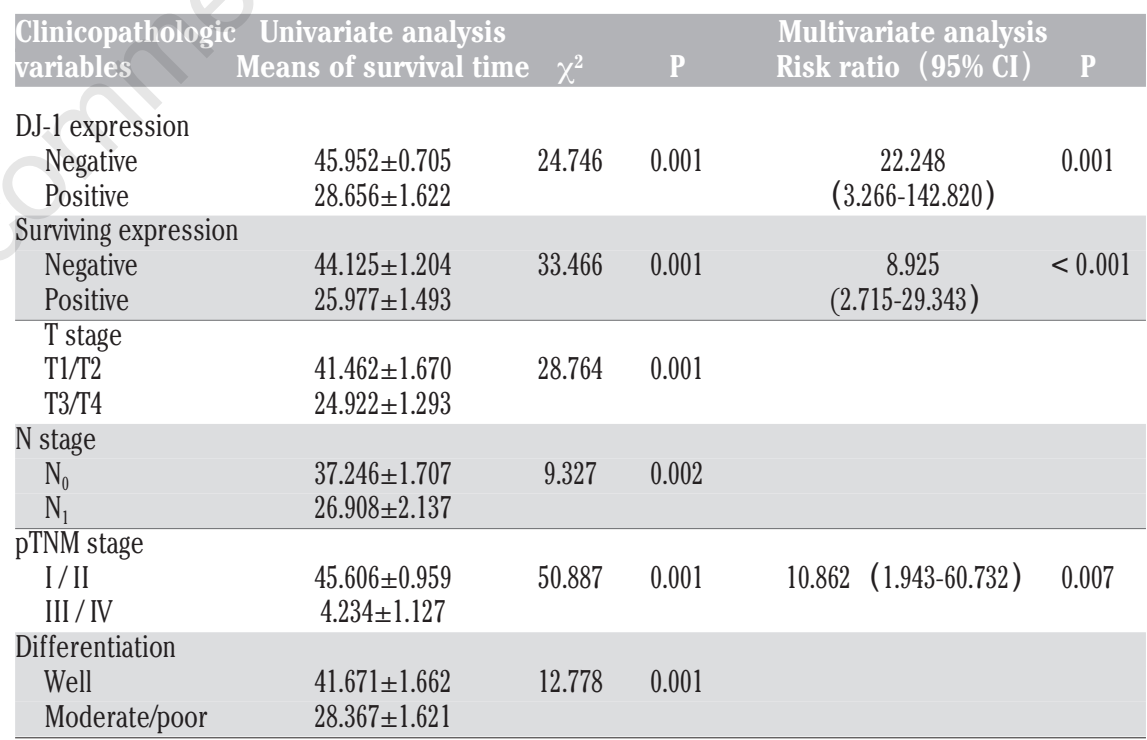

pTNM, pathological tumor-node-metastasis.

Table 5. Analysis of survival among DJ-1/survivin subgroups.

\begin{tabular}{|c|c|c|c|c|}
\hline Subgroups & n & Survival rate & $x^{2}$ & $\mathbf{P}$ \\
\hline DJ-1+/survivin $^{+}$ & 44 & $20.5 \%$ & 46.506 & 0.000 \\
\hline DJ-1+/survivin ${ }^{-}$ & 15 & $33.3 \%$ & & \\
\hline DJ-1-/survivin $^{+}$ & 6 & $50.0 \%$ & & \\
\hline DJ-1-/survivin'- & 17 & $88.2 \%$ & & \\
\hline
\end{tabular}


found that the PI3K signal transduction pathway regulated survivin on both mRNA level and protein level. Under normal circumstances, PTEN inhibits survivin expression through PI3K/AKT pathways. When PTEN activity gets lost, the expression of survivin will increase and then promote cell proliferation and inhibit apoptosis. ${ }^{12}$ A further study showed that PTEN also stimulated P21 expression and then increased the expression of survivin. ${ }^{13}$ We presume therefore that there are some regulation mechanisms between DJ-1 and survivin that function through PI3K/Akt/mTOR pathways.

In the subgroups study, we found that patients with DJ-1+/survivin+ had the poorest prognosis than other groups, while patients with DJ-1-/survivin- may indicate a better prognosis and longer survival (Table 5). Although a lot of researches advocating DJ-1 or survivin as potential biomarkers already have been performed, oncogenesis is a complex process involving multiple oncogenes and signal pathways; the combination use of oncogenes as cancer marker may be more persuasive than the use of single molecular marker. Our results indicate that combination of DJ-1 and survivin may be better biomarker for prognosis of laryngeal cancer.

\section{Conclusions}

Considered all together, our findings show that DJ-1 may regulate survivin expression as an upstream gene and the expressions of two genes are closely related to clinicopathologic parameters of laryngeal cancer. By Cox regression analysis, both of them are independent prognostic predictors for DFS of laryngeal cancer. DJ-1 and survivin could be used as potential biomarker for LSCC progression and prognosis.

\section{References}

1. Chu EA, Kim YJ. Laryngeal cancer: diagnosis and preoperative work-up. Otolaryngol Clin North Am 2008;41:673-95.

2. Bonifati V, Rizzu P, van Baren MJ, Schaap 0, Breedveld GJ, Krieger E, et al. Mutations in the DJ-1 gene associated with autosomal recessive early-onset parkinsonism. Science 2003;299:256-9.

3. Kim RH, Peters M, Jang Y, Shi W, Pintilie
M, Fletcher GC, et al. DJ-1, a novel regulator of the tumor suppressor PTEN. Cancer Cell 2005;7:263-73.

4. Shi W, Zhang X, Pintilie M, Ma N, Miller N, Banerjee D, et al. Dysregulated PTEN-PKB and negative receptor status in human breast cancer. Int J Cancer 2003;104:195203.

5. Le Naour F, Misek DE, Krause MC, Deneux L, Giordano TJ, Scholl S, et al. Proteomicsbased identification of RS/DJ-1 as a novel circulating tumor antigen in breast cancer. Clin Cancer Res 2001;7:3328-35.

6. MacKeigan JP, Clements CM, Lich JD, Pope RM, Hod Y,Ting JP. Proteomic profiling drug-induced apoptosis in non-small cell lung carcinoma: identification of RS/DJ-1 and RhoGDIalpha. Cancer Res 2003;63:6928-34.

7. Pennati M, Folini M, Zaffaroni N. Targeting survivin in cancer therapy: fulfilled promises and open questions. Carcinogenesis 2007;28:1133-9.

8. Duffy MJ, O'Donovan N, Brennan DJ, Gallagher WM,Ryan BM. Survivin: a promising tumor biomarker. Cancer Lett 2007;249:49-60.

9. Shen J, Liu J, Long Y, Miao Y, Su M, Zhang Q, et al. Knockdown of survivin expression by siRNAs enhances chemosensitivity of prostate cancer cells and attenuates its tumorigenicity. Acta Biochim Biophys Sin (Shanghai) 2009;41:223-30.

10. Andersen MH, Svane IM, Becker JC, Straten PT. The universal character of the tumor-associated antigen survivin. Clin Cancer Res 2007;13:5991-4.

11. Carter BZ, Milella M, Altieri DC, Andreeff M. Cytokine-regulated expression of survivin in myeloid leukemia. Blood 2001;97: 2784-90.

12. Suzuki A, Hayashida M, Ito T, Kawano H, Nakano T, Miura M, et al. Survivin initiates cell cycle entry by the competitive interaction with Cdk4/p16(INK4a) and Cdk2/cyclin E complex activation. Oncogene 2000;19:3225-34.

13. Wu RC, Li X, Schonthal AH. Transcriptional activation of p21WAF1 by PTEN /MMAC1 tumor suppressor. Mol Cell Biochem 2000;203:59-71.

14. Suzuki A, Ito T, Kawano H, Hayashida M, Hayasaki Y, Tsutomi Y, et al. Survivin initiates procaspase 3/p21 complex formation as a result of interaction with $\mathrm{Cdk} 4$ to resist Fas-mediated cell death. Oncogene 2000;19:1346-53.

15. Shen Z, Jiang Z, Ye D, Xiao B, Zhang X,
Guo J. Growth inhibitory effects of DJ-1small interfering RNA on laryngeal carcinoma Hep-2 cells. Med Oncol 2010 [Epub ahead of print].

16. Zhu XL, Wen WP, Lei WB, Chai LP, Hou WJ, Wen $\mathrm{YH}$, et al. DJ-1 expression in laryngeal squamous cell carcinoma and its relationship with tumor recurrence and metastasis. Zhonghua Er Bi Yan Hou Tou Jing Wai Ke Za Zhi 2010;45:497-501.

17. Erkanli S, Bolat F, Kayaselcuk F, Demirhan B, Kuscu E. COX-2 and survivin are overexpressed and positively correlated in endometrial carcinoma. Gynecol Oncol 2007;104:320-5.

18. Arnouk H, Merkley MA, Podolsky RH, Stoppler H, Santos C, Alvarez M, et al. Characterization of Molecular Markers Indicative of Cervical Cancer Progression. Proteomics Clin Appl 2009;3:516-27.

19. Wu F, Liang YQ, Huang ZM. The expression of DJ-1 gene in human hepatocellular carcinoma and its relationship with tumor invasion and metastasis. Zhonghua Gan Zang Bing Za Zhi 2009;17:203-6.

20. Landon MR, Lieberman RL, Hoang QQ, Ju $\mathrm{S}$, Caaveiro JM, Orwig SD, et al. Detection of ligand binding hot spots on protein surfaces via fragment-based methods: application to DJ-1 and glucocerebrosidase. J Comput Aided Mol Des. 2009 [Epub ahead of print].

21. Kawasaki H, Toyoda M, Shinohara H, Okuda J, Watanabe I, Yamamoto T, et al. Expression of survivin correlates with apoptosis, proliferation, and angiogenesis during human colorectal tumorigenesis. Cancer 2001;91:2026-32.

22. Wang Y, Kong W, Xiong X, Zhang S, Sun D. The expressions of survivin and VEGF in squamous cell carcinoma of larynx and the correlation between the two marks. Lin Chuang Er Bi Yan Hou Ke Za Zhi 2005;19:838-41.

23. Dong Y, Sui L, Watanabe Y, Sugimoto K, Tokuda M. Survivin expression in laryngeal squamous cell carcinomas and its prognostic implications. Anticancer Res 2002;22:2377-83.

24. Sitaram RT, Cairney CJ, Grabowski P, Keith WN, Hallberg B, Ljungberg B, et al. The PTEN regulator DJ-1 is associated with hTERT expression in clear cell renal cell carcinoma. Int J Cancer 2009;125:78390 . 